\title{
Quasilinear elliptic problems with combined critical Sobolev-Hardy terms
}

\author{
Yuanyuan Li · Bernhard Ruf · Qianqiao Guo . \\ Pengcheng Niu
}

Received: date / Accepted: date

\begin{abstract}
In this paper, by investigating the effect of the subcritical terms and the coefficients of the singular terms, some existence results for quasilinear elliptic problems involving combined critical Sobolev-Hardy terms are obtained via variational methods.
\end{abstract}

Keywords quasilinear elliptic problem · Hardy potential · critical Sobolev-Hardy term · existence $\cdot$ variational method

Mathematics Subject Classification (2000) $35 \mathrm{~J} 20 \cdot 35 \mathrm{~J} 47 \cdot 35 \mathrm{~J} 66$

\section{Introduction}

In this paper, we are concerned with the existence of solutions for the following quasilinear elliptic problem:

$$
\left\{\begin{aligned}
-\Delta_{p} u & =\mu \frac{|u|^{p-2} u}{|x|^{p}}+K(x) \frac{|u|^{p^{*}(s)-2} u}{|x|^{s}}+Q(x) \frac{|u|^{p^{*}(t)-2} u}{\left|x-x_{0}\right|^{t}}+f(x, u) & & \text { in } \Omega, \\
u & =0 & & \text { on } \partial \Omega
\end{aligned}\right.
$$

The project is supported by National Natural Science Foundation of China (Grant No. 11001221, 10871157) and Specialized Research Fund for the Doctoral Program of Higher Education (No. 200806990032).

Y. Li

Department of Applied Mathematics; Key Laboratory of Space Applied Physics and Chemistry, Ministry of Education, Northwestern Polytechnical University, Xi'an, Shaanxi, 710129, P. R. China

Dipartimento di Matematica, Università degli Studi di Milano, Via Saldini 50, 20133 Milano, Italy

E-mail: liyuanyuan@mail.nwpu.edu.cn

B. Ruf (凶)

Dipartimento di Matematica, Università degli Studi di Milano, Via Saldini 50, 20133 Milano, Italy

E-mail: ruf@mat.unimi.it

Q. Guo · P. Niu

Department of Applied Mathematics; Key Laboratory of Space Applied Physics and Chemistry, Ministry of Education, Northwestern Polytechnical University, Xi' an, Shaanxi, 710129, P. R. China

E-mail: gqianqiao@nwpu.edu.cn

P. Niu

E-mail: pengchengniu@nwpu.edu.cn 
where $\Omega$ is a smooth bounded domain in $\mathbb{R}^{N}(N \geq 3)$, and $-\Delta_{p} u$ denotes the $p$-Laplace operator defined as $-\Delta_{p} u=-\operatorname{div}\left(|\nabla u|^{p-2} \nabla u\right)$, with $1<p<N$. The parameter $\mu$ satisfies $0 \leq \mu<\bar{\mu}:=\left(\frac{N-p}{p}\right)^{p}$, the well-known Hardy constant. Furthermore, we assume that the points 0 and $x_{0}$ lie in $\Omega$, with $x_{0} \neq 0$. The exponents of the singular terms satisfy $0<$ $s, t<p$, and the exponents $p^{*}(s)$ and $p^{*}(t)$ of the corresponding nonlinearities are the critical exponents with respect to the Sobolev-Hardy embeddings into weighted $L^{q}$ spaces: $W^{1, p}(\Omega) \subset L^{p^{*}(s)}\left(\Omega,|x|^{-s} d x\right)$, with $p^{*}(s)=\frac{p(N-s)}{N-p}$. Finally, $K(x)$ and $Q(x)$ are continuous nonnegative functions, and $f(x, u)$ is a subcritical perturbation. Notice that for $s=0$ we get $p^{*}(0)=p^{*}:=\frac{N p}{N-p}$, the critical Sobolev exponent, while for $s=p$ we get $p^{*}(s)=p$, i.e. we have the Hardy embedding.

It is well known that if $u \in W_{0}^{1, p}(\Omega)$ is a nonzero critical point of the energy functional associated to (1)

$$
\begin{aligned}
J(u)= & \frac{1}{p} \int_{\Omega}\left(|\nabla u|^{p}-\mu \frac{|u|^{p}}{|x|^{p}}\right) d x-\frac{1}{p^{*}(s)} \int_{\Omega} K(x) \frac{|u|^{p^{*}(s)}}{|x|^{s}} d x-\frac{1}{p^{*}(t)} \int_{\Omega} Q(x) \frac{|u|^{p^{*}(t)}}{\left|x-x_{0}\right|^{t}} d x \\
& -\int_{\Omega} F(x, u) d x
\end{aligned}
$$

where $F(x, u)=\int_{0}^{u} f(x, s) d s$, then $u$ is a nontrivial weak solution for problem (1).

Elliptic equations with critical growth terms have received wide attention in recent years. In a pioneering work, Pohozaev [24] asserted that if $\Omega$ is a star-shaped domain with respect to the origin, then there is no nontrivial solution for the following elliptic problem

$$
\left\{\begin{array}{cl}
-\Delta u=|u|^{2^{*}-2} u & \text { in } \Omega, \\
u=0 & \text { on } \partial \Omega .
\end{array}\right.
$$

However, lower order terms can reverse this situation. Indeed, Brezis and Nirenberg showed in their seminal paper [2] that there exist positive solutions for the perturbed elliptic problem with critical Sobolev growth

$$
\left\{\begin{array}{cl}
-\Delta u=\lambda u+|u|^{2^{*}-2} u & \text { in } \Omega, \\
u=0 & \text { on } \partial \Omega,
\end{array}\right.
$$

by verifying that for appropriate values of $\lambda$ the energy of the corresponding energy functional is less than a certain non-compactness level. Generalizations of this result can be found in [15], and multiplicity results for equations with a variable coefficient in CaoNoussair [5]. Later, the existence of positive solutions for equation (3) was established on certain non-starshaped domains, see Coron [8] and Ding [9].

Struwe [27] obtained a global compactness result for problem (4), and showed that the lack of compactness is due to the invariance of the $H_{0}^{1}$ - norm and $L^{2^{*}}$ - norm under the rescaling $u \mapsto u_{r}=r^{\frac{N-2}{2}} u(r(\cdot))$ and the existence of nontrivial entire solutions for the limiting problem (3) in $\mathbb{R}^{N}$. In particular, he gave an asymptotic characterization of all PS-sequences for (4), showing that compactness is lost only due to the formation of "standard bubbles" given by the rescaled solutions of the asymptotic equation (3).

Jannelli [20] considered the following doubly-critical semilinear elliptic problem with a Hardy term

$$
\begin{cases}-\Delta u-\mu \frac{u}{|x|^{2}}=u^{2^{*}-1}+\lambda u & \text { in } \Omega \\ u=0 & \text { on } \partial \Omega\end{cases}
$$


and proved the existence of solutions. This problem was also discussed by Ferrero-Gazzola [11], by Cao-Peng [6], Ruiz-Willem [25] and by Cao-Han [3]. Guo et al. [18] and Chen [7] considered the equation with a critical Sobolev-Hardy nonlinearity, and showed the existence and multiplicity of nontrivial solutions. Other interesting results about related problems can be found in Ghoussoub-Ekeland [9] and in the references therein.

Quasilinear elliptic problems involving the $p$-Laplacian and nonlinearities with the corresponding critical Sobolev growth were studied by Silva-Xavier [26]. Ghoussoub-Yuan [16] considered such equations with a critical Sobolev-Hardy term, and proved existence results for positive solutions and sign-changing solutions.

As for quasilinear elliptic problems with a singular Hardy term and a critical SobolevHardy nonlinearity, the following problem

$$
\begin{cases}-\Delta_{p} u-\mu \frac{|u|^{p-2} u}{|x|^{p}}=b(x) \frac{|u|^{p^{*}(s)-2} u}{|x|^{s}}+h(x, u) & \text { in } \Omega \\ u=0 & \text { on } \partial \Omega\end{cases}
$$

has been investigated by several authors, where $b(x)$ is a continuous non-negative function and $h(x, u)$ is a subcritical perturbation, see Kang [21], Filipucci-Pucci-Robert [12]; in [23], Liang et al. considered problem (6) for $b(x) \equiv 1$ and derived the existence of infinitely many small solutions by using the concentration compactness principle and a symmetric mountain pass theorem.

Concerning problems with multiple critical Sobolev-Hardy terms centered in different points, we mention Gao-Peng [13], who studied problem (1) with $p=2$ and obtained some existence results by investigating the limit behavior of the PS-sequences for the corresponding energy functional.

For $p$-Laplace equations involving multiple critical Sobolev-Hardy terms as well as Hardy terms there has been little research up to now. We point out that concerning problem (1), Li et al. [22] have established the complete asymptotic description for any PS-sequence $\left(u_{n}\right)$ of the associated energy functional (2). They show that in the presence of multiple critical Sobolev-Hardy terms the possible concentration of PS-sequences is localized in the singularities of the nonlinear terms, that is, concentration may only occur in the points 0 and $x_{0}$, and the associated levels of non-compactness for the functional (2) are explicit, and there is compactness away from these levels. The aim of the present paper is to provide sufficient conditions on the coefficients $K(x), Q(x)$ and the lower order term $f(x, u)$ in order to guarantee that there exist minimax levels of the associated functional (2) which lie below the lowest non-compactness level, thus regaining compactness and hence the existence of critical points. Depending on the values $K(0)$ and $Q\left(x_{0}\right)$ the concentration with lowest energy occurs in 0 or $x_{0}$. The two cases require separate treatment, and the results are stated in Theorems 3.1 and 4.1. We remark that the situations are not symmetric: indeed, in Sect. 3 and Sect. 4, the following two limiting problems

$$
-\Delta_{p} u-\mu \frac{|u|^{p-2} u}{|x|^{p}}=\frac{|u|^{p^{*}(s)-2} u}{|x|^{s}}, \text { in } \mathbb{R}^{N} \backslash\{0\}
$$

and

$$
-\Delta_{p} u=\frac{|u|^{p^{*}(t)-2} u}{\left|x-x_{0}\right|^{t}}, \text { in } \mathbb{R}^{N} \backslash\left\{x_{0}\right\}
$$

are used, respectively. We note that the accurate form of the solutions for the first limiting problem is not clear, different from the second one, which leads to some clear differences between the proofs in Sect. 3 and in Sect. 4. 
Finally, in Sect. 5 we will give conditions on $K(x)$ and $Q(x)$ under which, due to a Pohozaev type identity, there do not exist nontrivial solutions for the equation

$$
\begin{cases}-\Delta_{p} u=\mu \frac{|u|^{p-2} u}{|x|^{p}}+K(x) \frac{|u|^{p^{*}(s)-2} u}{|x|^{s}}+Q(x) \frac{|u|^{p^{*}(t)-2} u}{\left|x-x_{0}\right|^{t}} & \text { in } \Omega, \\ u=0 & \text { on } \partial \Omega .\end{cases}
$$

It is clear that our approach can be generalized to the case of several critical SobolevHardy terms which are centered in different points in $\Omega$.

\section{Hypotheses and Functional Setup}

Throughout this paper, we assume that the function $f(x, u): \Omega \times \mathbb{R} \rightarrow \mathbb{R}$ is a Carathéodory function of the form

$$
f(x, u)=a(x)|u|^{p-2} u+g(x, u),
$$

with

$$
\begin{gathered}
g(x, u)>0 \text { and } g(x, u)=o\left(u^{p-1}\right) \text { as } u \rightarrow 0^{+} \text {, uniformly in } x ; \\
g(x, u)=o\left(u^{\gamma-1}\right) \text { as } u \rightarrow \infty, \text { uniformly in } x, \text { where } p<\gamma<p^{*} .
\end{gathered}
$$

In the sequel, $B(x, r)$ denotes a ball of radius $r$ with center in $x$, and $d x$ in integrals is omitted for convenience.

We also need to assume that the first eigenvalue of the operator $-\Delta_{p}-\frac{\mu}{|x|^{p}}-a(x)$ is positive, that is we assume that there exists an $\alpha>0$ such that

$$
\int_{\Omega}\left(|\nabla u|^{p}-\mu \frac{|u|^{p}}{|x|^{p}}-a(x)|u|^{p}\right) \geq \alpha \int_{\Omega}|u|^{p}, \text { for all } u \in W_{0}^{1, p}(\Omega) .
$$

Set

$$
0<a(0)<\lambda_{1}(\mu), 0<a\left(x_{0}\right)<\lambda_{1}(\mu),
$$

where

$$
\lambda_{1}(\mu)=\inf _{u \in W_{0}^{1, p}(\Omega) \backslash\{0\}} \frac{\int_{\Omega}|\nabla u|^{p}-\mu \frac{|u|^{p}}{|x|^{p}}}{\int_{\Omega}|u|^{p}}
$$

is the first eigenvalue of the positive operator $\left(-\Delta_{p}-\frac{\mu}{|x|^{p}}\right)$.

Define

$$
\|u\|_{W_{0}^{1, p}(\Omega)}:=\left[\int_{\Omega}|\nabla u|^{p}-\mu \frac{|u|^{p}}{|x|^{p}}\right]^{\frac{1}{p}} .
$$

By Hardy's inequality (see [14]), we can conclude that the above norm is equivalent to the usual norm

$$
\|u\|=\left(\int_{\Omega}|\nabla u|^{p} d x\right)^{\frac{1}{p}}
$$

in $W_{0}^{1, p}(\Omega)$.

Next, we recall the following standard compactness definition: 
Definition 2.1 A $C^{1}$-functional $E$ defined on Banach space $X$ satisfies the Palais-Smale condition at the level $c\left((\mathrm{PS})_{c}\right.$ in short), if every Palais-Smale (PS in short) sequence $\left\{u_{n}\right\} \subset$ $X$ has a convergent subsequence. And a sequence $\left\{u_{n}\right\}$ is a PS sequence for $E$ if $\left\{u_{n}\right\}$ satisfies $E\left(u_{n}\right) \rightarrow c$ and $E^{\prime}\left(u_{n}\right) \rightarrow 0$ as $n \rightarrow \infty$.

In the sequel, let $E=J$ (see (2)) and $X=W_{0}^{1, p}(\Omega)$.

To state the main results, we need the following notations:

$$
\begin{gathered}
F_{\mu, K(0)}^{\infty}(u)=\frac{1}{p} \int_{\Omega}|\nabla u|^{p}-\mu \frac{|u|^{p}}{|x|^{p}}-\frac{1}{p^{*}(s)} \int_{\Omega} K(0) \frac{|u|^{p^{*}(s)}}{|x|^{s}} \\
F_{0, Q\left(x_{0}\right)}^{\infty}(u)=\frac{1}{p} \int_{\Omega}|\nabla u|^{p}-\frac{1}{p^{*}(t)} \int_{\Omega} Q\left(x_{0}\right) \frac{|u|^{p^{*}(t)}}{\left|x-x_{0}\right|^{t}}
\end{gathered}
$$

Furthermore, set

$$
A_{\mu, s}=\inf _{u \in W_{0}^{1, p}(\Omega) \backslash\{0\}} \frac{\int_{\Omega}|\nabla u|^{p}-\mu \frac{|u|^{p}}{|x|^{p}}}{\left(\int_{\Omega} \frac{|u|^{p^{*}(s)}}{|x|^{s}}\right)^{\frac{p}{p^{*}(s)}}}
$$

and

$$
A_{0, t}=\inf _{u \in W_{0}^{1, p}(\Omega) \backslash\{0\}} \frac{\int_{\Omega}|\nabla u|^{p}}{\left(\int_{\Omega} \frac{|u|^{p^{*}(t)}}{\left|x-x_{0}\right|^{t}}\right)^{\frac{p}{p^{*}(t)}}} .
$$

We also assume that

$$
K(0)>0 \text { and } Q\left(x_{0}\right)>0
$$

Denote

$$
K^{*}=\frac{p-s}{p(N-s)} K(0)^{\frac{N-p}{s-p}} A_{\mu, s}^{\frac{N-s}{p-s}},
$$

and

$$
Q^{*}=\frac{p-t}{p(N-t)} Q\left(x_{0}\right)^{\frac{N-p}{t-p}} A_{0, t}^{\frac{N-t}{p-t}} .
$$

Then we have the following key lemma due to Li et al. [22].

Lemma 2.1 The functional J given by (2) satisfies the $(P S)_{c}$ condition for all

$$
c<\min \left\{K^{*}, Q^{*}\right\}
$$


3 Existence result when $K^{*} \leq Q^{*}$

Before giving the main theorem in this section, we state the following lemma.

Lemma 3.1 ([21]). Assume $1<p<N, 0 \leq s<p$ and $0 \leq \mu<\bar{\mu}$. Then the problem

$$
\begin{cases}-\Delta_{p} u-\mu \frac{|u|^{p-2} u}{|x|^{p}}=\frac{|u|^{p^{*}(s)-2} u}{|x|^{s}} & \text { in } \mathbb{R}^{N} \backslash\{0\}, \\ u>0 & \text { in } \mathbb{R}^{N} \backslash\{0\}, \\ u \in D^{1, p}\left(\mathbb{R}^{N}\right) & \end{cases}
$$

has radially symmetric ground states

$$
\bar{V}_{\varepsilon}(x)=\varepsilon^{-\frac{N-p}{p}} U_{p, \mu}\left(\frac{x}{\varepsilon}\right)=\varepsilon^{-\frac{N-p}{p}} U_{p, \mu}\left(\frac{|x|}{\varepsilon}\right), \forall \varepsilon>0,
$$

satisfying

$$
\int_{\mathbb{R}^{N}}\left(\left|\nabla \bar{V}_{\varepsilon}(x)\right|^{p}-\mu \frac{\left|\bar{V}_{\varepsilon}(x)\right|^{p}}{|x|^{p}}\right)=\int_{\mathbb{R}^{N}} \frac{\left|\bar{V}_{\varepsilon}(x)\right|^{p^{*}(s)}}{|x|^{s}}=\left(A_{\mu, s}\right)^{\frac{N-s}{p-s}},
$$

where $U_{p, \mu}(x)=U_{p, \mu}(|x|)$ is the unique radial solution for the problem (14) satisfying

$$
U_{p, \mu}(1)=\left(\frac{(N-s)(\bar{\mu}-\mu)}{N-p}\right)^{\frac{1}{p^{*}(s)-p}} .
$$

Moreover, $U_{p, \mu}(x)$ also has the following properties,

$$
\begin{aligned}
& \lim _{r \rightarrow 0} r^{a(\mu)} U_{p, \mu}(r)=c_{1}>0, \\
& \lim _{r \rightarrow+\infty} r^{b(\mu)} U_{p, \mu}(r)=c_{2}>0, \\
& \lim _{r \rightarrow 0} r^{a(\mu)+1} U_{p, \mu}^{\prime}(r)=c_{1} a(\mu) \geq 0, \\
& \lim _{r \rightarrow+\infty} r^{b(\mu)+1} U_{p, \mu}^{\prime}(r)=c_{2} b(\mu)>0,
\end{aligned}
$$

where $c_{1}$ and $c_{2}$ are positive constants depending on $p$ and $N, a(\mu)$ and $b(\mu)$ are zeros of the function

$$
f(\tau)=(p-1) \tau^{p}-(N-p) \tau^{p-1}+\mu, \tau \geq 0,0 \leq \mu<\bar{\mu}
$$

satisfying

$$
0 \leq a(\mu)<\frac{N-p}{p}<b(\mu) \leq \frac{N-p}{p-1}
$$

Remark. After a direct calculation, we deduce that $\tau_{\min }=\frac{N-p}{p}$ is the only minimal point of $f(\tau)$. Furthermore, $f^{\prime}(\tau)<0$ for $0<\tau<\tau_{\min }$ and $f^{\prime}(\tau)>0$ for $\tau>\tau_{\min }$. Thus, we infer that

$$
\tau_{\min }<\frac{N}{p} \leq b(\mu) \Longleftrightarrow f\left(\frac{N}{p}\right) \leq f(b(\mu))=0 \Longleftrightarrow 0<\mu \leq \frac{N^{p-1}\left(N-p^{2}\right)}{p^{p}} .
$$

Furthermore, by (16) we know that $b(\mu)>\frac{N}{p}$ implies $N>p^{2}$. 
Theorem 3.1 Suppose $K^{*} \leq Q^{*}$ and (8)-(10) hold. Moreover, if $b(\mu)>\frac{N}{p}$, then $K(x)=$ $K(0)+O\left(|x|^{\beta}\right)$ as $|x| \rightarrow 0$ holds with some $\beta>p$; if $b(\mu) \leq \frac{N}{p}$, then $K(x)=K(0)+$ $O\left(|x|^{\beta}\right)$ as $|x| \rightarrow 0$ with some $\beta \geq p$ and

there exists an open nonempty subset $\Omega_{0} \subset \Omega$, such that $0 \in \Omega_{0}$

and $\lim _{u \rightarrow \infty} \frac{F(x, u)}{u^{l}}=+\infty$ uniformly with respect to $x \in \Omega_{0}$,

where $l>\Lambda=\max \left\{p, \frac{p(2 N-p-p b(\mu))}{N-p}\right\}$. Then there is at least one nontrivial solution $u \in$ $W_{0}^{1, p}(\Omega)$ of the problem $(1)$.

In order to prove Theorem 3.1, we need the following key lemma:

Lemma 3.2 Under the assumptions of Theorem 3.1, there exists a function $u \in W_{0}^{1, p}(\Omega)$ satisfying

$$
\sup _{\tau \geq 0} J(\tau u)<K^{*}
$$

Proof Set $\rho>0$ small enough such that $B(0, \rho) \subset \Omega, \varphi(x) \in C_{0}^{\infty}(\Omega), 0 \leq \varphi(x) \leq 1, \varphi(x)=$ 1 for $|x| \leq \frac{\rho}{2}, \varphi(x)=0$ for $|x| \geq \rho$. Let

$$
v_{\varepsilon}(x)=\varphi(x) \bar{V}_{\varepsilon}(x), \varepsilon>0 .
$$

The following estimates are from [19] and [21].

$$
\begin{gathered}
\left\|v_{\mathcal{\varepsilon}}\right\|_{W_{0}^{1, p}(\Omega)}^{p}=\left(A_{\mu, s}\right)^{\frac{N-s}{p-s}}+O\left(\varepsilon^{b(\mu) p+p-N}\right), \\
\int_{\Omega} \frac{\left|v_{\varepsilon}\right|^{p^{*}(s)}}{|x|^{s}}=\left(A_{\mu, s}\right)^{\frac{N-s}{p-s}}+O\left(\varepsilon^{b(\mu) p^{*}(s)+s-N}\right), \\
\int_{\Omega}\left|v_{\varepsilon}\right|^{q} \geq \begin{cases}c \varepsilon^{N+\left(1-\frac{N}{p}\right) q}, & q>\frac{N}{b(\mu)} \\
c \varepsilon^{N+\left(1-\frac{N}{p}\right) q}|\ln \varepsilon|, & q=\frac{N}{b(\mu)} \\
c \varepsilon^{q\left(b(\mu)+1-\frac{N}{p}\right)}, & q<\frac{N}{b(\mu)}\end{cases}
\end{gathered}
$$

Especially, when $q=p$, we have

$$
\int_{\Omega}\left|v_{\varepsilon}\right|^{p} \geq \begin{cases}c \varepsilon^{p}, & p>\frac{N}{b(\mu)} \\ c \varepsilon^{p}|\ln \varepsilon|, & p=\frac{N}{b(\mu)} \\ c \varepsilon^{p-N+p b(\mu)}, & p<\frac{N}{b(\mu)}\end{cases}
$$

Observe that

$$
\begin{aligned}
\int_{\Omega} K(x) \frac{\left|v_{\varepsilon}\right|^{p^{*}(s)}}{|x|^{s}}= & K(0) \int_{\mathbb{R}^{N}} \frac{\left|\bar{V}_{\varepsilon}\right|^{p^{*}(s)}}{|x|^{s}}+\int_{\Omega}(K(x)-K(0))|\varphi|^{p^{*}(s)} \frac{\left|\bar{V}_{\varepsilon}\right|^{p^{*}(s)}}{|x|^{s}} \\
& -K(0) \int_{\mathbb{R}^{N}}\left(1-|\varphi|^{p^{*}(s)}\right) \frac{\left|\bar{V}_{\varepsilon}\right|^{p^{*}(s)}}{|x|^{s}}
\end{aligned}
$$




$$
\begin{aligned}
= & K(0) \int_{\mathbb{R}^{N}} \frac{\left|U_{p, \mu}\right|^{p^{*}(s)}}{|x|^{s}}+\varepsilon^{-(N-s)} \int_{\Omega}(K(x)-K(0))|\varphi|^{p^{*}(s)} \frac{\left|U_{p, \mu}\left(\frac{x}{\varepsilon}\right)\right|^{p^{*}(s)}}{|x|^{s}} \\
& -\varepsilon^{-(N-s)} K(0) \int_{\mathbb{R}^{N}}\left(1-|\varphi|^{p^{*}(s)}\right) \frac{\left|U_{p, \mu}\left(\frac{x}{\varepsilon}\right)\right|^{p^{*}(s)}}{|x|^{s}} \\
:= & K(0) \int_{\mathbb{R}^{N}} \frac{\left|U_{p, \mu}\right|^{p^{*}(s)}}{|x|^{s}}+I_{1}+I_{2} .
\end{aligned}
$$

Now we calculate $I_{1}$ and $I_{2}$, respectively. First for $\delta_{0}>0$ small enough we deduce that

$$
\begin{aligned}
\left|I_{1}\right| \leq & \left|\varepsilon^{-(N-s)} \int_{\Omega}(K(x)-K(0)) \frac{\left|U_{p, \mu}\left(\frac{x}{\varepsilon}\right)\right|^{p^{*}(s)}}{|x|^{s}}\right| \\
\leq & c \varepsilon^{-(N-s)} \int_{\Omega \cap\left\{|x| \leq \delta_{0}\right\}}|x|^{\beta} \frac{\left(\left|\frac{x}{\varepsilon}\right|^{a(\mu)}+\left|\frac{x}{\varepsilon}\right|^{b(\mu)}\right)^{-p^{*}(s)}}{|x|^{s}} \\
& +c \varepsilon^{-(N-s)} \int_{\Omega \cap\left\{|x| \geq \delta_{0}\right\}}\left|\frac{x}{\varepsilon}\right|^{-b(\mu) p^{*}(s)} \frac{1}{|x|^{s}} \\
\leq & c \varepsilon^{-(N-s)} \int_{0}^{\delta_{0}} r^{\beta+N-1-s}\left(\left|\frac{r}{\varepsilon}\right|^{a(\mu)}+\left|\frac{r}{\varepsilon}\right|^{b(\mu)}\right)^{-p^{*}(s)}+c \varepsilon^{p^{*}(s) b(\mu)-N+s} \\
\leq & c \varepsilon^{-(N-s)} \int_{0}^{\varepsilon^{-1}} \delta_{0} \varepsilon^{\beta+N-s} t^{\beta+N-1-s}\left(t^{a(\mu)}+t^{b(\mu)}\right)^{-p^{*}(s)}+c \varepsilon^{p^{*}(s) b(\mu)-N+s} \\
\leq & c \varepsilon^{\beta} \int_{0}^{\varepsilon^{-1} \delta_{0}} t^{\beta+N-1-s-a(\mu) p^{*}(s)}\left(1+t^{b(\mu)-a(\mu)}\right)^{-p^{*}(s)}+c \varepsilon^{p^{*}(s) b(\mu)-N+s .} .
\end{aligned}
$$

If $\beta<p^{*}(s) b(\mu)-N+s$, then

$$
\beta+N-1-s-a(\mu) p^{*}(s)-p^{*}(s)(b(\mu)-a(\mu))<-1 .
$$

We conclude that

$$
I_{1}=O\left(\varepsilon^{\beta}\right)
$$

If $\beta=p^{*}(s) b(\mu)-N+s$, then

$$
\beta+N-1-s-a(\mu) p^{*}(s)-p^{*}(s)(b(\mu)-a(\mu))=-1 .
$$

So we get

$$
I_{1}=O\left(\varepsilon^{p^{*}(s) b(\mu)-N+s}|\ln \varepsilon|\right)
$$

If $\beta>p^{*}(s) b(\mu)-N+s$, then

$$
\beta+N-1-s-a(\mu) p^{*}(s)-p^{*}(s)(b(\mu)-a(\mu))>-1 .
$$

We have

$$
I_{1}=O\left(\varepsilon^{p^{*}(s) b(\mu)-N+s}\right) .
$$

Therefore we obtain that

$$
I_{1}= \begin{cases}O\left(\varepsilon^{\beta}\right), & \beta<p^{*}(s) b(\mu)-N+s \\ O\left(\varepsilon^{p^{*}(s) b(\mu)-N+s}|\ln \varepsilon|\right), & \beta=p^{*}(s) b(\mu)-N+s \\ O\left(\varepsilon^{p^{*}(s) b(\mu)-N+s}\right), & \beta>p^{*}(s) b(\mu)-N+s\end{cases}
$$


Again by $b(\mu)>\frac{N-p}{p}$, it is not difficult to derive that

$$
\begin{aligned}
\left|I_{2}\right| & =\left|\varepsilon^{-(N-s)} K(0) \int_{\mathbb{R}^{N}}\left(1-|\varphi|^{p^{*}(s)}\right) \frac{\left|U_{p, \mu}\left(\frac{x}{\varepsilon}\right)\right|^{p^{*}(s)}}{|x|^{s}}\right| \\
& \leq \varepsilon^{-(N-s)} K(0) \int_{\mathbb{R}^{N} \backslash|x| \geq \frac{\rho}{2}} \frac{\left|U_{p, \mu}\left(\frac{x}{\varepsilon}\right)\right|^{*^{*}(s)}}{|x|^{s}} \\
& \leq c \varepsilon^{b(\mu) p^{*}(s)-N+s} \int_{\mathbb{R}^{N} \backslash|x| \geq \frac{\rho}{2}}|x|^{-b(\mu) p^{*}(s)-s} \\
& \leq c \varepsilon^{b(\mu) p^{*}(s)-N+s} \int_{\frac{\rho}{2}}^{\infty} r^{N-1-b(\mu) p^{*}(s)-s} \\
& \leq c \varepsilon^{p^{*}(s) b(\mu)-N+s},
\end{aligned}
$$

thus

$$
I_{2}=O\left(\varepsilon^{p^{*}(s) b(\mu)-N+s}\right) .
$$

Therefore, inserting (24) and (25) into (23), we obtain

$$
\begin{gathered}
\int_{\Omega} K(x) \frac{\left|v_{\varepsilon}\right|^{p^{*}(s)}}{|x|^{s}} \\
=K(0)\left(A_{\mu, s}\right)^{\frac{N-s}{p-s}}+ \begin{cases}O\left(\varepsilon^{\beta}\right), & \beta<p^{*}(s) b(\mu)-N+s, \\
O\left(\varepsilon^{p^{*}(s) b(\mu)-N+s}|\ln \varepsilon|\right), & \beta=p^{*}(s) b(\mu)-N+s, \\
O\left(\varepsilon^{p^{*}(s) b(\mu)-N+s}\right), & \beta>p^{*}(s) b(\mu)-N+s .\end{cases}
\end{gathered}
$$

Now we consider the following functions

$$
\begin{aligned}
g(\tau)=J\left(\tau v_{\varepsilon}\right)= & \frac{\tau^{p}}{p} \int_{\Omega}\left(\left|\nabla v_{\varepsilon}\right|^{p}-\mu \frac{\left|v_{\varepsilon}\right|^{p}}{|x|^{p}}\right)-\frac{\tau^{p^{*}(s)}}{p^{*}(s)} \int_{\Omega} K(x) \frac{\left|v_{\varepsilon}\right|^{p^{*}(s)}}{|x|^{s}} \\
& -\frac{\tau^{p^{*}(t)}}{p^{*}(t)} \int_{\Omega} Q(x) \frac{\left|v_{\varepsilon}\right|^{p^{*}(t)}}{\left|x-x_{0}\right|^{t}}-\int_{\Omega} F\left(x, \tau v_{\varepsilon}\right)
\end{aligned}
$$

and

$$
\bar{g}(\tau)=\frac{\tau^{p}}{p}\left\|v_{\varepsilon}\right\|_{W_{0}^{1, p}(\Omega)}^{p}-\frac{\tau^{p^{*}(s)}}{p^{*}(s)} \int_{\Omega} K(x) \frac{\left|v_{\varepsilon}\right|^{p^{*}(s)}}{|x|^{s}} .
$$

Using the fact that

$$
\max _{\tau \geq 0}\left(\frac{\tau^{p}}{p} B_{1}-\frac{\tau^{p^{*}(s)}}{p^{*}(s)} B_{2}\right)=\frac{p-s}{p(N-s)} B_{1}^{\frac{N-s}{p-s}} B_{2}^{-\frac{N-p}{p-s}}, B_{1}>0, B_{2}>0
$$

by (19) and (26) we calculate that

$$
\begin{aligned}
\max _{\tau \geq 0} \bar{g}(\tau) & =\frac{p-s}{p(N-s)}\left\|v_{\varepsilon}\right\|_{W_{0}^{1, p}(\Omega)}^{\frac{p(N-s)}{p-s}}\left(\int_{\Omega} K(x) \frac{\left|v_{\varepsilon}\right|^{p^{*}(s)}}{|x|^{s}}\right)^{-\frac{N-p}{p-s}} \\
& =\frac{p-s}{p(N-s)} K(0)^{\frac{N-p}{s-p}}\left(A_{\mu, s}\right)^{\frac{N-s}{p-s}}+O\left(\varepsilon^{b(\mu) p+p-N}\right)
\end{aligned}
$$




$$
+ \begin{cases}O\left(\varepsilon^{\beta}\right), & \beta<p^{*}(s) b(\mu)-N+s \\ O\left(\varepsilon^{p^{*}(s) b(\mu)-N+s}|\ln \varepsilon|\right), & \beta=p^{*}(s) b(\mu)-N+s \\ O\left(\varepsilon^{p^{*}(s) b(\mu)-N+s}\right), & \beta>p^{*}(s) b(\mu)-N+s\end{cases}
$$

By (8)-(10), (12) and (17), we can select nonnegative constants $a_{1}$ and $a_{2}$, such that

$$
f(x, u) \geq a_{1} u^{p-1}+a_{2} u^{l-1}
$$

for $x \in B(0, \rho)$ and $u \geq 0$ if $\rho>0$ small enough, where $0 \leq a_{2} \leq a_{1}<\lambda_{1}(\mu), a_{2}=$ 0 if $b(\mu)>\frac{N}{p}, a_{2}>0$ and $\Lambda<l<\gamma$ if $b(\mu) \leq \frac{N}{p}$. Combining this with (21) and (22), for arbitrary $\tau \in(0,+\infty)$ we have

$$
\int_{\Omega} F\left(x, \tau v_{\varepsilon}\right) \geq\left\{\begin{array}{ll}
c \varepsilon^{p}, & p>\frac{N}{b(\mu)} \\
c \varepsilon^{p}|\ln \varepsilon|, & p=\frac{N}{b(\mu)} \\
c \varepsilon^{p-N+p b(\mu)}, & p<\frac{N}{b(\mu)}
\end{array}+ \begin{cases}c \varepsilon^{l\left(b(\mu)+1-\frac{N}{p}\right)}, & p<l<\frac{N}{b(\mu)} \\
c \varepsilon^{N+\left(1-\frac{N}{p}\right) l}|\ln \varepsilon|, & l=\frac{N}{b(\mu)}, \\
c \varepsilon^{N+\left(1-\frac{N}{p}\right) l}, & \frac{N}{b(\mu)}<l<\gamma\end{cases}\right.
$$

where $c=c(\tau)$.

Since

$$
\lim _{\tau \rightarrow+\infty} g(\tau)=-\infty
$$

and by (12) we have

$$
g(\tau)>0 \text { as } \tau \rightarrow 0
$$

So $\sup _{\tau \geq 0} g(\tau)$ is attained for some $0<\tau_{0}<+\infty$. Together with (27) and (28), we obtain that

$$
\begin{aligned}
& g(\tau) \leq g\left(\tau_{0}\right) \\
& \leq \max _{\tau \geq 0} \bar{g}(\tau)-\int_{\Omega} F\left(x, \tau_{0} v_{\varepsilon}\right) \\
& \leq \frac{p-s}{p(N-s)} K(0)^{\frac{N-p}{s-p}}\left(A_{\mu, s}\right)^{\frac{N-s}{p-s}}+O\left(\varepsilon^{b(\mu) p+p-N}\right) \\
& + \begin{cases}O\left(\varepsilon^{\beta}\right), & \beta<p^{*}(s) b(\mu)-N+s, \\
O\left(\varepsilon^{p^{*}(s) b(\mu)-N+s}|\ln \varepsilon|\right), & \beta=p^{*}(s) b(\mu)-N+s, \\
O\left(\varepsilon^{p^{*}(s) b(\mu)-N+s}\right), & \beta>p^{*}(s) b(\mu)-N+s,\end{cases} \\
& -\left\{\begin{array}{ll}
c \varepsilon^{p}, & p>\frac{N}{b(\mu)} \\
c \varepsilon^{p}|\ln \varepsilon|, & p=\frac{N}{b(\mu)} \\
c \varepsilon^{p-N+p b(\mu)}, & p<\frac{N}{b(\mu)}
\end{array}- \begin{cases}c \varepsilon^{l\left(b(\mu)+1-\frac{N}{p}\right)}, & p<l<\frac{N}{b(\mu)}, \\
c \varepsilon^{N+\left(1-\frac{N}{p}\right) l}|\ln \varepsilon|, & l=\frac{N}{b(\mu)}, \\
c \varepsilon^{N+\left(1-\frac{N}{p}\right) l}, & \frac{N}{b(\mu)}<l<\gamma .\end{cases} \right.
\end{aligned}
$$

Now we distinguish two cases: that is, $b(\mu)>\frac{N}{p}$ and $b(\mu) \leq \frac{N}{p}$.

(C1). If $b(\mu)>\frac{N}{p}$, then by (15) we get

$$
p^{*}(s) b(\mu)-N+s>b(\mu) p+p-N>p,
$$

and

$$
\beta>p .
$$

For $\varepsilon$ small enough, by (29) we deduce that

$$
g\left(\tau_{0}\right) \leq \frac{p-s}{p(N-s)} K(0)^{\frac{N-p}{s-p}}\left(A_{\mu, s}\right)^{\frac{N-s}{p-s}}-O\left(\varepsilon^{p}\right)
$$




$$
<\frac{p-s}{p(N-s)} K(0)^{\frac{N-p}{s-p}}\left(A_{\mu, s}\right)^{\frac{N-s}{p-s}}
$$

(C2). If $b(\mu) \leq \frac{N}{p}$, then by (15) and $l>\Lambda$ we have

$$
\begin{gathered}
l>\frac{p(2 N-p-p b(\mu))}{N-p} \geq \frac{N}{b(\mu)}, \\
\beta \geq p=N+p\left(1-\frac{N}{p}\right)>N+l\left(1-\frac{N}{p}\right),
\end{gathered}
$$

and

$$
p^{*}(s) b(\mu)-N+s>b(\mu) p+p-N>N+l\left(1-\frac{N}{p}\right) .
$$

For $\varepsilon$ small enough, by (29) we get that

$$
\begin{aligned}
g\left(\tau_{0}\right) & \leq \frac{p-s}{p(N-s)} K(0)^{\frac{N-p}{s-p}}\left(A_{\mu, s}\right)^{\frac{N-s}{p-s}}-O\left(\varepsilon^{N+l\left(1-\frac{N}{p}\right)}\right) \\
& <\frac{p-s}{p(N-s)} K(0)^{\frac{N-p}{s-p}}\left(A_{\mu, s}\right)^{\frac{N-s}{p-s}} .
\end{aligned}
$$

Hence (18) holds and the proof of the lemma is completed by taking $u=v_{\varepsilon}$.

Proof of Theorem 3.1. Set

$$
c=\inf _{\psi \in \Psi} \sup _{\tau \in[0,1]} J(\psi(\tau)),
$$

where

$$
\Psi=\left\{\psi \in C\left([0,1], W_{0}^{1, p}(\Omega)\right), \psi(0)=0, \psi(1)=\psi_{0} \in W_{0}^{1, p}(\Omega)\right\},
$$

and $\psi_{0}$ satisfies $J\left(\tau \psi_{0}\right) \leq 0$ for all $\tau \geq 1$. By (8)-(10), there exist positive constants $c_{1}$ and $c_{2}$ such that

$$
g(x, u) \leq c_{1} u^{p-1}+c_{2} u^{\gamma-1} .
$$

Thus by (8) we have

$$
F(x, u) \leq \frac{1}{p} a(x)|u|^{p}+\frac{1}{p} c_{1}|u|^{p}+\frac{c_{2}}{\gamma}|u|^{\gamma}
$$

for a.e. $x \in \Omega$. Hence for all $u \in W_{0}^{1, p}(\Omega)$, by (11) and Sobolev-Hardy's inequality, we derive that

$$
\begin{aligned}
J(u) \geq & \frac{1}{p} \int_{\Omega}\left(|\nabla u|^{p}-\mu \frac{|u|^{p}}{|x|^{p}}\right)-\frac{1}{p^{*}(s)} \int_{\Omega} K(x) \frac{|u|^{p^{*}(s)}}{|x|^{s}}-\frac{1}{p^{*}(t)} \int_{\Omega} Q(x) \frac{|u|^{p^{*}(t)}}{\left|x-x_{0}\right|^{t}} \\
& -\int_{\Omega} \frac{1}{p} a(x)|u|^{p}+\frac{1}{p} c_{1}|u|^{p}+\frac{c_{2}}{\gamma}|u|^{\gamma} \\
\geq & \left(A-B\|u\|_{W_{0}^{1, p}(\Omega)}^{p^{*}(s)-p}-C\|u\|_{W_{0}^{1, p}(\Omega)}^{p^{*}(t)-p}-D\|u\|_{W_{0}^{1, p}(\Omega)}^{\gamma-p}\right)\|u\|_{W_{0}^{1, p}(\Omega)}^{p},
\end{aligned}
$$

where $A, B, C$ and $D$ are all positive constants, which implies that there exist $\rho_{0}>0$ and $c^{\prime}>0$ such that

$$
\left.J\right|_{\partial B\left(0, \rho_{0}\right)}>c^{\prime}>0 .
$$

Notice that

$$
J\left(\tau v_{\varepsilon}\right) \rightarrow-\infty \text { as } \tau \rightarrow+\infty,
$$


thus there exists $\tau_{1}>0$ such that

$$
\left\|\tau_{1} v_{\varepsilon}\right\|_{W_{0}^{1, p}(\Omega)}>\rho_{0}
$$

and

$$
J\left(\tau_{1} v_{\varepsilon}\right)<0 .
$$

Therefore by using the Mountain Pass Lemma, there exists a sequence $\left\{u_{n}\right\} \subset W_{0}^{1, p}(\Omega)$ such that

$$
J\left(u_{n}\right) \rightarrow c, J^{\prime}\left(u_{n}\right) \rightarrow 0 \text { in }\left(W_{0}^{1, p}(\Omega)\right)^{-1} .
$$

By Lemma 3.2, we know that

$$
0<c^{\prime} \leq c \leq \sup _{\tau \in[0,1]} J\left(\tau \tau_{1} v_{\varepsilon}\right) \leq \sup _{\tau \geq 0} J\left(\tau v_{\varepsilon}\right)<\frac{p-s}{p(N-s)} K(0)^{\frac{N-p}{s-p}}\left(A_{\mu, s}\right)^{\frac{N-s}{p-s}}=K^{*} .
$$

By Lemma 2.1, $\left\{u_{n}\right\}$ has a subsequence, still denoted by $\left\{u_{n}\right\}$, such that

$$
u_{n} \rightarrow u \text { strongly in } W_{0}^{1, p}(\Omega) \text {. }
$$

Hence combining this with (30) we deduce that $u \in W_{0}^{1, p}(\Omega)$ is a critical point of the functional $J$ and is also a nontrivial solution for the problem (1).

\section{Existence result when $Q^{*}<K^{*}$}

First we introduce the following lemma which is from [16].

Lemma 4.1 Suppose $1<p<N, 0 \leq t<p$. Then the following holds:

(i) $A_{0, t}$ is independent of $\Omega$;

(ii) $A_{0, t}$ is attained when $\Omega=\mathbb{R}^{N}$ by the functions

$$
y_{\varepsilon}(x)=\left(\varepsilon(N-t)\left(\frac{N-p}{p-1}\right)^{p-1}\right)^{\frac{N-p}{p(p-t)}}\left(\varepsilon+\left|x-x_{0}\right|^{\frac{p-t}{p-1}}\right)^{\frac{p-N}{p-t}}
$$

for some $\varepsilon>0$. Moreover the functions $y_{\varepsilon}(x)$ solve the equation

$$
-\Delta_{p} u=\frac{|u|^{p^{*}(t)-2} u}{\left|x-x_{0}\right|^{t}} \text { in } \mathbb{R}^{N} \backslash\left\{x_{0}\right\},
$$

and satisfy

$$
\int_{\mathbb{R}^{N}}\left|\nabla y_{\mathcal{\varepsilon}}\right|^{p}=\int_{\mathbb{R}^{N}} \frac{\left|y_{\mathcal{\varepsilon}}\right|^{p^{*}(t)}}{\left|x-x_{0}\right|^{t}}=\left(A_{0, t}\right)^{\frac{N-t}{p-t}}
$$

Theorem 4.1 Suppose $Q^{*}<K^{*}$ and (8)-(10) hold. Moreover, if $N>p^{2}$, then $Q(x)=Q\left(x_{0}\right)+$ $O\left(\left|x-x_{0}\right|^{\sigma}\right)$ as $\left|x-x_{0}\right| \rightarrow 0$ holds with some $\sigma>p$; if $N \leq p^{2}$, then $Q(x)=Q\left(x_{0}\right)+O(\mid x-$ $\left.\left.x_{0}\right|^{\sigma}\right)$ as $\left|x-x_{0}\right| \rightarrow 0$ with some $\sigma \geq p$ and

there exists an open nonempty subset $\Omega_{0} \subset \Omega$, such that $x_{0} \in \Omega_{0}$

$$
\text { and } \lim _{u \rightarrow \infty} \frac{F(x, u)}{u^{l}}=+\infty \text { uniformly with respect to } x \in \Omega_{0} \text {, }
$$

where $l>\frac{p N(p-1)-p(N-p)}{(p-1)(N-p)}$. Then there is at least one nontrivial solution $u \in W_{0}^{1, p}(\Omega)$ of the problem (1). 
Now we give the following key lemma which is very useful for the proof of Theorem 4.1.

Lemma 4.2 Under the assumptions of Theorem 4.1, there exists a function $u \in W_{0}^{1, p}(\Omega)$ such that

$$
\sup _{\tau \geq 0} J(\tau u)<Q^{*}
$$

Proof Let

$$
\begin{gathered}
C_{\varepsilon}=\left(\varepsilon(N-t)\left(\frac{N-p}{p-1}\right)^{p-1}\right)^{\frac{N-p}{p(p-t)}}, \\
U_{\varepsilon}(x)=\frac{y_{\varepsilon}(x)}{C_{\varepsilon}} .
\end{gathered}
$$

Set $\varphi(x) \in C_{0}^{\infty}(\Omega), 0 \leq \varphi(x) \leq 1, \varphi(x)=1$ for $\left|x-x_{0}\right| \leq \frac{R}{2}, \varphi(x)=0$ for $\left|x-x_{0}\right| \geq$ $R$, where $B\left(x_{0}, R\right) \subset \Omega$. Denote

$$
\begin{gathered}
u_{\varepsilon}(x)=\varphi(x) U_{\varepsilon}(x), \text { for all } \varepsilon>0, \\
w_{\varepsilon}(x)=\frac{u_{\varepsilon}(x)}{\left(\int_{\Omega} \frac{\left|u_{\varepsilon}\right|^{*}(t)}{\left|x-x_{0}\right|^{t}}\right)^{\frac{1}{p^{*}(t)}}},
\end{gathered}
$$

such that

$$
\int_{\Omega} \frac{\left|w_{\varepsilon}\right|^{p^{*}(t)}}{\left|x-x_{0}\right|^{t}}=1 .
$$

Then we can obtain the following results by the methods used in [16],

$$
\begin{gathered}
\left\|w_{\mathcal{E}}\right\|_{W_{0}^{1, p}(\Omega)}^{p}=A_{0, t}+O\left(\varepsilon^{\frac{N-p}{p-t}}\right), \\
\int_{\Omega}\left|w_{\varepsilon}\right|^{q} \geq \begin{cases}c \varepsilon^{\frac{q(N-p)}{p(p-t)}}, & q<\frac{N(p-1)}{N-p}, \\
c \varepsilon^{\frac{q(N-p)}{p(p-t)}}|\ln \varepsilon|, & q=\frac{N(p-1)}{N-p}, \\
c \varepsilon^{\frac{(p-1)(p N-q(N-p))}{p(p-t)}}, & q>\frac{N(p-1)}{N-p} .\end{cases}
\end{gathered}
$$

Especially, when $q=p$, we have that

$$
\int_{\Omega}\left|w_{\varepsilon}\right|^{p} \geq \begin{cases}c \varepsilon^{\frac{N-p}{p-t}}, & p^{2}>N \\ c \varepsilon^{\frac{N-p}{p-t}}|\ln \varepsilon|, & p^{2}=N \\ c \varepsilon^{\frac{p(p-1)}{p-t}}, & p^{2}<N\end{cases}
$$

Observing that $w_{\varepsilon}$ concentrates on $x=x_{0}$ when $\varepsilon>0$ small enough, we can easily calculate that

$$
\int_{\Omega} \frac{\left|w_{\varepsilon}\right|^{p}}{|x|^{p}} \geq \begin{cases}c \varepsilon^{\frac{N-p}{p-t}}, & p^{2}>N \\ c \varepsilon^{\frac{N-p}{p-t}}|\ln \varepsilon|, & p^{2}=N \\ c \varepsilon^{\frac{p(p-1)}{p-t}}, & p^{2}<N\end{cases}
$$


Notice that

$$
\begin{aligned}
\int_{\Omega}\left|x-x_{0}\right|^{k} \frac{\left|u_{\varepsilon}\right|^{*}(t)}{\left|x-x_{0}\right|^{t}} & =O(1)+\int_{\left|x-x_{0}\right| \leq R}\left|x-x_{0}\right|^{k-t}\left(\varepsilon+\left|x-x_{0}\right|^{\frac{p-t}{p-1}}\right)^{\frac{p(N-t)}{t-p}} \\
& =O(1)+c \int_{0}^{R} r^{k-t+N-1}\left(\varepsilon+r^{\frac{p-t}{p-1}}\right)^{\frac{p(N-t)}{t-p}} \\
& =O(1)+c \varepsilon^{\frac{k(p-1)-(N-t)}{p-t}} \int_{0}^{R \varepsilon^{-\frac{p-1}{p-t}}} r^{k-t+N-1}\left(1+r^{\frac{p-t}{p-1}}\right)^{\frac{p(N-t)}{t-p}} .
\end{aligned}
$$

If $k<\frac{N-t}{p-1}$, then

$$
k-t+N-1+\frac{p-t}{p-1} \frac{p(N-t)}{t-p}<-1 .
$$

We conclude that

$$
\int_{\Omega}\left|x-x_{0}\right|^{k} \frac{\left|u_{\varepsilon}\right|^{p^{*}(t)}}{\left|x-x_{0}\right|^{t}}=O\left(\varepsilon^{\frac{k(p-1)-(N-t)}{p-t}}\right) .
$$

If $k=\frac{N-t}{p-1}$, then

$$
k-t+N-1+\frac{p-t}{p-1} \frac{p(N-t)}{t-p}=-1 .
$$

So we get

$$
\int_{\Omega}\left|x-x_{0}\right|^{k} \frac{\left|u_{\varepsilon}\right|^{p^{*}(t)}}{\left|x-x_{0}\right|^{t}}=O(|\ln \varepsilon|)
$$

If $k>\frac{N-t}{p-1}$, then

$$
k-t+N-1+\frac{p-t}{p-1} \frac{p(N-t)}{t-p}>-1 .
$$

We have

$$
\int_{\Omega}\left|x-x_{0}\right|^{k} \frac{\left|u_{\varepsilon}\right|^{p^{*}(t)}}{\left|x-x_{0}\right|^{t}}=O(1)
$$

Therefore we obtain that

$$
\int_{\Omega}\left|x-x_{0}\right|^{k} \frac{\left|u_{\varepsilon}\right|^{p^{*}(t)}}{\left|x-x_{0}\right|^{t}}= \begin{cases}O\left(\varepsilon^{\frac{k(p-1)-(N-t)}{p-t}}\right), & k<\frac{N-t}{p-1} \\ O(|\ln \varepsilon|), & k=\frac{N-t}{p-1} \\ O(1), & k>\frac{N-t}{p-1}\end{cases}
$$

and hence

$$
\int_{\Omega}\left|x-x_{0}\right|^{k} \frac{\left|w_{\varepsilon}\right|^{p^{*}(t)}}{\left|x-x_{0}\right|^{t}}= \begin{cases}O\left(\varepsilon^{\frac{k(p-1)}{p-t}}\right), & k<\frac{N-t}{p-1}, \\ O\left(\varepsilon^{\frac{N-t}{p-t}}|\ln \varepsilon|\right), & k=\frac{N-t}{p-1}, \\ O\left(\varepsilon^{\frac{N-t}{p-t}}\right), & k>\frac{N-t}{p-1} .\end{cases}
$$

By (37), for $R>0$ small enough and $x \in B\left(x_{0}, R\right)$ we infer that

$$
\int_{\Omega} Q(x) \frac{\left|w_{\varepsilon}\right|^{p^{*}(t)}}{\left|x-x_{0}\right|^{t}} \geq \int_{\Omega} Q\left(x_{0}\right) \frac{\left|w_{\varepsilon}\right|^{p^{*}(t)}}{\left|x-x_{0}\right|^{t}}-c \int_{\Omega}\left|x-x_{0}\right|^{\sigma} \frac{\left|w_{\varepsilon}\right|^{p^{*}(t)}}{\left|x-x_{0}\right|^{t}}
$$




$$
=Q\left(x_{0}\right)- \begin{cases}O\left(\varepsilon^{\frac{\sigma(p-1)}{p-t}}\right), & \sigma<\frac{N-t}{p-1} \\ O\left(\varepsilon^{\frac{N-t}{p-t}}|\ln \varepsilon|\right), & \sigma=\frac{N-t}{p-1} \\ O\left(\varepsilon^{\frac{N-t}{p-t}}\right), & \sigma>\frac{N-t}{p-1}\end{cases}
$$

Now we consider the following function

$$
\begin{aligned}
h(\tau)=J\left(\tau w_{\varepsilon}\right)= & \frac{\tau^{p}}{p} \int_{\Omega}\left(\left|\nabla w_{\varepsilon}\right|^{p}-\mu \frac{\left|w_{\varepsilon}\right|^{p}}{|x|^{p}}\right)-\frac{\tau^{p^{*}(s)}}{p^{*}(s)} \int_{\Omega} K(x) \frac{\left|w_{\varepsilon}\right|^{p^{*}(s)}}{|x|^{s}} \\
& -\frac{\tau^{p^{*}(t)}}{p^{*}(t)} \int_{\Omega} Q(x) \frac{\left|w_{\varepsilon}\right|^{p^{*}(t)}}{\left|x-x_{0}\right|^{t}}-\int_{\Omega} F\left(x, \tau w_{\varepsilon}\right) .
\end{aligned}
$$

By (8)-(10), (12) and (31), we can find nonnegative constants $a_{1}$ and $a_{2}$, such that

$$
f(x, u) \geq a_{1} u^{p-1}+a_{2} u^{l-1}
$$

for $x \in B\left(x_{0}, R\right)$ and $u \geq 0$ if $R>0$ small enough, where $0 \leq a_{2} \leq a_{1}<\lambda_{1}(\mu), a_{2}=0$ if $N>$ $p^{2}, a_{2}>0$ and $\frac{p N(p-1)-p(N-p)}{(p-1)(N-p)}<l<\gamma$ if $N \leq p^{2}$. Combining this with (34) and (35), for arbitrary $\tau \in(0, \infty)$ we have that

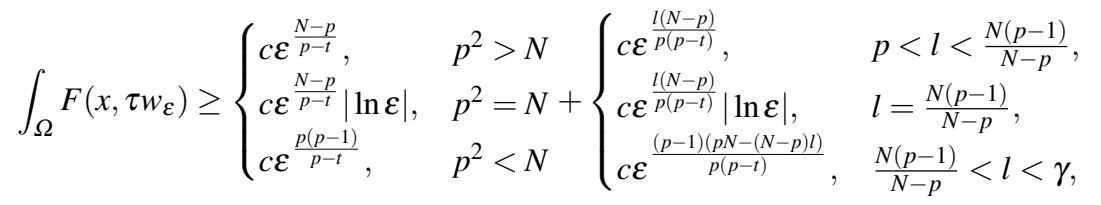

where $c=c(\tau)$.

Since

$$
\lim _{\tau \rightarrow+\infty} h(\tau)=-\infty
$$

by (12) we get

$$
h(\tau)>0 \text { as } \tau \rightarrow 0 .
$$

So $\sup h(\tau)$ is attained for some $0<\tau_{0}<+\infty$. Together with (33), (36), (38) and (39), we $\tau \geq 0$
calculate that

$$
\begin{aligned}
& h(\tau) \leq h\left(\tau_{0}\right) \\
& \leq \frac{\tau_{0}^{p}}{p}\left\|w_{\varepsilon}\right\|_{W_{0}^{1, p}(\Omega)}^{2}-\frac{\tau_{0}^{p^{*}(t)}}{p^{*}(t)} \int_{\Omega} Q(x) \frac{\left|w_{\varepsilon}\right|^{p^{*}(t)}}{\left|x-x_{0}\right|^{t}}-\int_{\Omega} F\left(x, \tau_{0} w_{\varepsilon}\right) \\
& \leq \frac{p-t}{p(N-t)} Q\left(x_{0}\right)^{\frac{N-p}{t-p}}\left(A_{0, t}\right)^{\frac{N-t}{p-t}}+O\left(\varepsilon^{\frac{N-p}{p-t}}\right)+ \begin{cases}O\left(\varepsilon^{\frac{\sigma(p-1)}{p-t}}\right), & \sigma<\frac{N-t}{p-1}, \\
O\left(\varepsilon^{\frac{N-t}{p-t}}|\ln \varepsilon|\right), & \sigma=\frac{N-t}{p-1}, \\
O\left(\varepsilon^{\frac{N-t}{p-t}}\right), & \sigma>\frac{N-t}{p-1},\end{cases}
\end{aligned}
$$

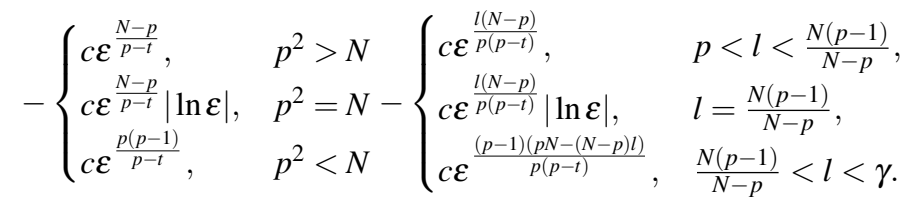

Now we distinguish two cases: that is, $N \leq p^{2}$ and $N>p^{2}$. 
(C1). If $N \leq p^{2}$, noticing that

$$
l>\frac{N p(p-1)-p(N-p)}{(p-1)(N-p)} \geq \frac{N(p-1)}{N-p},
$$

we have

$$
\frac{\sigma(p-1)}{p-t} \geq \frac{N-p}{p-t}>\frac{(p-1)(p N-(N-p) l)}{p(p-t)},
$$

and

$$
\frac{N-t}{p-t}>\frac{N-p}{p-t} \text {. }
$$

By (40), we deduce for $\varepsilon$ small enough,

$$
\begin{aligned}
h\left(\tau_{0}\right) & <\frac{p-t}{p(N-t)} Q\left(x_{0}\right)^{\frac{N-p}{t-p}}\left(A_{0, t}\right)^{\frac{N-t}{p-t}}-c \varepsilon^{\frac{(p-1)(p N-(N-p) l)}{p(p-t)}} \\
& <\frac{p-t}{p(N-t)} Q\left(x_{0}\right)^{\frac{N-p}{t-p}}\left(A_{0, t}\right)^{\frac{N-t}{p-t}} .
\end{aligned}
$$

(C2). If $N>p^{2}$, then

$$
\frac{N-t}{p-t}>\frac{N-p}{p-t}>\frac{p(p-1)}{p-t},
$$

and

By (40), for $\varepsilon$ small enough we derive that

$$
\frac{\sigma(p-1)}{p-t}>\frac{p(p-1)}{p-t} \text {. }
$$

$$
\begin{aligned}
h\left(\tau_{0}\right) & <\frac{p-t}{p(N-t)} Q\left(x_{0}\right)^{\frac{N-p}{t-p}}\left(A_{0, t}\right)^{\frac{N-t}{p-t}}-c \varepsilon^{\frac{p(p-1)}{p-t}} \\
& <\frac{p-t}{p(N-t)} Q\left(x_{0}\right)^{\frac{N-p}{t-p}}\left(A_{0, t}\right)^{\frac{N-t}{p-t}} .
\end{aligned}
$$

Hence (32) holds and we end the proof by taking $u=w_{\varepsilon}$.

Proof of Theorem 4.1. Set

$$
c=\inf _{\psi \in \Psi} \sup _{\tau \in[0,1]} J(\psi(\tau)),
$$

where

$$
\Psi=\left\{\psi \in C\left([0,1], W_{0}^{1, p}(\Omega)\right), \psi(0)=0, \psi(1)=\psi_{0} \in W_{0}^{1, p}(\Omega)\right\},
$$

and $\psi_{0}$ satisfies $J\left(\tau \psi_{0}\right) \leq 0$ for all $\tau \geq 1$. Then similarly to the proof of Theorem 3.1, we infer that the functional $J(u)$ satisfies all the assumptions of the Mountain Pass Lemma. Hence there exists a sequence $\left\{u_{n}\right\} \subset W_{0}^{1, p}(\Omega)$ such that

$$
J\left(u_{n}\right) \rightarrow c, J^{\prime}\left(u_{n}\right) \rightarrow 0 \text { in }\left(W_{0}^{1, p}(\Omega)\right)^{-1} .
$$

By Lemma 4.2, we deduce that

$$
0<c^{\prime} \leq c \leq \sup _{\tau \in[0,1]} J\left(\tau \tau_{1} w_{\varepsilon}\right) \leq \sup _{\tau \geq 0} J\left(\tau w_{\varepsilon}\right)<\frac{p-t}{p(N-t)} Q\left(x_{0}\right)\left(A_{0, t}\right)^{\frac{N-t}{p-t}}=Q^{*},
$$

where $c^{\prime}$ and $\tau_{1}$ are defined in the proof of Theorem 3.1. Then by Lemma 2.1, $\left\{u_{n}\right\}$ has a subsequence, still denoted by $\left\{u_{n}\right\}$, such that

$$
u_{n} \rightarrow u \text { strongly in } W_{0}^{1, p}(\Omega) .
$$

Therefore together with (41) we deduce that $u$ is a critical point of $J$ and is also a nontrivial solution for the problem (1). 


\section{A nonexistence result}

In this section, we prove a nonexistence result for the problem (7).

Theorem 5.1 Assume $K(x), Q(x) \in C^{1}(\Omega)$ and $\Omega$ is a strictly star-sharped domain with respect to the origin in $\mathbb{R}^{N}$. If

$$
\langle x, \nabla K(x)\rangle \leq 0,\left\langle x, \nabla Q(x)-\frac{t Q(x)}{\left|x-x_{0}\right|^{2}}\left(x-x_{0}\right)\right\rangle+t Q(x) \leq 0, \text { for all } x \in \Omega,
$$

then there exists no nontrivial solution for the problem (7).

Proof Suppose that we have the necessary regularity in the following operations. Otherwise, we can use an approximation argument as in [17].

Set

$$
d(x, u)=\mu \frac{|u|^{p-2} u}{|x|^{p}}+K(x) \frac{|u|^{p^{*}(s)-2} u}{|x|^{s}}+Q(x) \frac{|u|^{p^{*}(t)-2} u}{\left|x-x_{0}\right|^{t}} .
$$

Then

$$
\begin{gathered}
D(x, u)=\int_{0}^{u} d(x, v) d v=\frac{1}{p} \mu \frac{|u|^{p}}{|x|^{p}}+\frac{1}{p^{*}(s)} K(x) \frac{|u|^{p^{*}(s)}}{|x|^{s}}+\frac{1}{p^{*}(t)} Q(x) \frac{|u|^{p^{*}(t)}}{\left|x-x_{0}\right|^{t}} \\
\left(\partial_{i} D\right)(x, u)=-\mu \frac{|u|^{p}}{|x|^{p+2}} x_{i}-\frac{s}{p^{*}(s)} K(x) \frac{|u|^{p^{*}(s)}}{|x|^{s+2}} x_{i}-\frac{t}{p^{*}(t)} Q(x) \frac{|u|^{p^{*}(t)}}{\left|x-x_{0}\right|^{t+2}}\left(x_{i}-x_{i 0}\right) \\
+\frac{1}{p^{*}(s)} \partial_{i} K(x) \frac{|u|^{p^{*}(s)}}{|x|^{s}}+\frac{1}{p^{*}(t)} \partial_{i} Q(x) \frac{|u|^{p^{*}(t)}}{\left|x-x_{0}\right|^{\mid}} \\
\partial_{i}(D(x, u))=\left(\partial_{i} D\right)(x, u)+d(x, u) \partial_{i} u .
\end{gathered}
$$

Multiplying equation (7) by $\langle x, \nabla u\rangle$ on both sides, we get

$$
-\int_{\Omega} \Delta_{p} u\langle x, \nabla u\rangle=\int_{\Omega} d(x, u)\langle x, \nabla u\rangle .
$$

An application of the divergence theorem yields that

$$
\begin{aligned}
\int_{\Omega} \Delta_{p} u\langle x, \nabla u\rangle & =\int_{\Omega} \operatorname{div}\left(|\nabla u|^{p-2} \nabla u\right)\langle x, \nabla u\rangle \\
& =\int_{\partial \Omega}|\nabla u|^{p}\langle x, v\rangle d S-\int_{\Omega}|\nabla u|^{p-2}\langle\nabla u, \nabla(x, \nabla u)\rangle \\
& =\int_{\partial \Omega}|\nabla u|^{p}\langle x, v\rangle d S-\int_{\Omega}|\nabla u|^{p}-\frac{1}{p} \int_{\Omega}\left\langle x, \nabla\left(|\nabla u|^{p}\right)\right\rangle \\
& =\int_{\partial \Omega}|\nabla u|^{p}\langle x, v\rangle d S-\int_{\Omega}|\nabla u|^{p}-\frac{1}{p} \int_{\partial \Omega}|\nabla u|^{p}\langle x, v\rangle d S+\frac{n}{p} \int_{\Omega}|\nabla u|^{p} \\
& =\frac{p-1}{p} \int_{\partial \Omega}|\nabla u|^{p}\langle x, v\rangle d S-\frac{p-n}{p} \int_{\Omega}|\nabla u|^{p}
\end{aligned}
$$

where $v$ is the outwards normal to $\partial \Omega$. 
Again by (45), (43), (44) and integrating by parts, we derive that

$$
\begin{aligned}
\int_{\Omega} d(x, u)\langle x, \nabla u\rangle= & -n \int_{\Omega} D(x, u)-\int_{\Omega} \sum_{i} x_{i}\left(\partial_{i} D\right)(x, u) \\
= & -\frac{n-p}{p} \int_{\Omega} \mu \frac{|u|^{p}}{|x|^{p}}-\frac{n-s}{p^{*}(s)} \int_{\Omega} K(x) \frac{|u|^{p^{*}(s)}}{|x|^{s}}-\frac{n-t}{p^{*}(t)} \int_{\Omega} Q(x) \frac{|u|^{p^{*}(t)}}{\left|x-x_{0}\right|^{t}} \\
& -\frac{1}{p^{*}(s)} \int_{\Omega}\langle x, \nabla K\rangle \frac{|u|^{p^{*}(s)}}{|x|^{s}}-\frac{1}{p^{*}(t)} \int_{\Omega}\langle x, \nabla Q\rangle \frac{|u|^{p^{*}(t)}}{\left|x-x_{0}\right|^{t}} \\
& +\frac{t}{p^{*}(t)} \int_{\Omega} Q(x) \frac{|u|^{p^{*}(t)}}{\left|x-x_{0}\right|^{t+2}}\left\langle x_{0}, x-x_{0}\right\rangle .
\end{aligned}
$$

Then inserting (47) and (48) into (46), we deduce that

$$
\begin{aligned}
& \frac{p-1}{p} \int_{\partial \Omega}|\nabla u|^{p}\langle x, v\rangle d S-\frac{p-n}{p} \int_{\Omega}|\nabla u|^{p}-\mu \frac{|u|^{p}}{|x|^{p}} \\
= & \frac{n-s}{p^{*}(s)} \int_{\Omega} K(x) \frac{|u|^{p^{*}(s)}}{|x|^{s}}+\frac{n-t}{p^{*}(t)} \int_{\Omega} Q(x) \frac{|u|^{p^{*}(t)}}{\left|x-x_{0}\right|^{t}} \\
& +\frac{1}{p^{*}(s)} \int_{\Omega}\langle x, \nabla K\rangle \frac{|u|^{p^{*}(s)}}{|x|^{S}}+\frac{1}{p^{*}(t)} \int_{\Omega}\langle x, \nabla Q\rangle \frac{|u|^{p^{*}(t)}}{\left|x-x_{0}\right|^{t}} \\
& -\frac{t}{p^{*}(t)} \int_{\Omega} Q(x) \frac{|u|^{p^{*}(t)}}{\left|x-x_{0}\right|^{t+2}}\left\langle x_{0}, x-x_{0}\right\rangle .
\end{aligned}
$$
that

On the other hand, multiplying the equation (7) by $u$ and integrating by parts, we obtain

$$
\int_{\Omega}|\nabla u|^{p}-\mu \frac{|u|^{p}}{|x|^{p}}=\int_{\Omega} K(x) \frac{|u|^{p^{*}(s)}}{|x|^{s}}+Q(x) \frac{|u|^{p^{*}(t)}}{\left|x-x_{0}\right|^{t}} .
$$

By (49) and (50), we have

$$
\begin{aligned}
& \frac{p-1}{p} \int_{\partial \Omega}|\nabla u|^{p}\langle x, v\rangle d S \\
= & \left(\frac{n-s}{p^{*}(s)}+\frac{p-n}{p}\right) \int_{\Omega} K(x) \frac{|u|^{p^{*}(s)}}{|x|^{s}}+\left(\frac{n-t}{p^{*}(t)}+\frac{p-n}{p}\right) \int_{\Omega} Q(x) \frac{|u|^{p^{*}(t)}}{\left|x-x_{0}\right|^{t}} \\
& +\frac{1}{p^{*}(s)} \int_{\Omega}\langle x, \nabla K\rangle \frac{|u|^{p^{*}(s)}}{|x|^{s}}+\frac{1}{p^{*}(t)} \int_{\Omega}\langle x, \nabla Q\rangle \frac{|u|^{p^{*}(t)}}{\left|x-x_{0}\right|^{t}} \\
& -\frac{t}{p^{*}(t)} \int_{\Omega} Q(x) \frac{|u|^{p^{*}(t)}}{\left|x-x_{0}\right|^{t+2}}\left\langle x_{0}, x-x_{0}\right\rangle,
\end{aligned}
$$

which is equivalent to

$$
\begin{aligned}
& \frac{p-1}{p} \int_{\partial \Omega}|\nabla u|^{p}\langle x, v\rangle d S \\
= & \frac{1}{p^{*}(s)} \int_{\Omega}\langle x, \nabla K\rangle \frac{|u|^{p^{*}(s)}}{|x|^{S}}+\frac{1}{p^{*}(t)} \int_{\Omega}\langle x, \nabla Q\rangle \frac{|u|^{p^{*}(t)}}{\left|x-x_{0}\right|^{t}}
\end{aligned}
$$




$$
-\frac{t}{p^{*}(t)} \int_{\Omega} Q(x) \frac{|u|^{p^{*}(t)}}{\left|x-x_{0}\right|^{t+2}}\left\langle x_{0}, x-x_{0}\right\rangle .
$$

If $\Omega$ is a strictly star-sharped domain with respect to the origin, then $\langle x, v\rangle>0$ on $\partial \Omega$. Thus by (42) and (51) we conclude that the problem (7) has no nontrivial solution.

\section{References}

1. Ambrosetti, A., Rabinowitz, H.: Dual varitional methods in critical point theory and applications. J. Funct. Anal. 14, 349-381 (1973)

2. Brezis, H., Nirenberg, L.: Positive solutions of nonlinear elliptic equations involving critical Sobolev exponent. Comm. Pure Appl. Math. 36, 437-477 (1983)

3. Cao, D.M., Han, P.G.: Solutions for semilinear elliptic equations with critical exponents and Hardy potential. J. Differ. Equ. 205, 521-537 (2004)

4. Cao, D.M., He, X.M., Peng, S.J.: Positive solutions for some singular critical growth nonlinear elliptic equations. Nonlinear Anal. TMA 60, 589-609 (2005)

5. Cao, D.M., Noussair, E.S.: Multiple positive and nodal solutions for semilinear elliptic problems with critical exponents. Indiana Univ. Math. J. 44, 1249-1271 (1995)

6. Cao, D.M., Peng, S.J.: A note on the sign-changing solutions to elliptic problems with critical Sobolev and Hardy terms. J. Differ. Equ. 193, 424-434 (2003)

7. Chen, J.: On a semilinear elliptic equation with singular term and Hardy-Sobolev critical growth, Math. Nachr. 280, No. 8, 838-850 (2007)

8. Coron, J.M.: Topologie et cas limite des injections de Sobolev. C. R. Acad. Sc. Paris 299 (Ser. I), 209-212 (1984)

9. Ding, W.Y.: Positive solution of $\Delta u+u^{\frac{N+2}{N-2}}=0$ on contractible domains, J. Partial Differ. Equ. 2, 83-88 (1989)

10. Ekeland, I., Ghoussoub, N.: Selected new aspects of the calculus of variations in the large. Bull. Amer. Math. Soc. 39, 207-265 (2002)

11. Ferrero, A., Gazzola, F.: Existence of solutions for singular critical growth semilinear elliptic equations. J. Differ. Equ. 177, 494-522 (2001)

12. Filippucci, R., Pucci, P., Robert, F.: On a $p$-Laplace equation with multiple critical nonlinearities, J. Math. Pures Appl. 91, 156-177 (2009)

13. Gao, W.L., Peng, S.J.: An elliptic equation with combined critical Sobolev-Hardy terms. Nonlinear Anal. TMA 65, 1595-1612 (2006)

14. Garcia Azorero, J.P., Peral Alonso, I.: Hardy inequalities and some critical elliptic and parabolic problems. J. Differ. Equ. 144, 441-476 (1998)

15. Gazzola, F., Ruf, B.: Lower-order perturbations of critical growth nonlinearties in semilinear elliptic equations. Adva. Differ. Equ. 2, 555-572 (1997)

16. Ghoussoub, N., Yuan, C.: Multiple solutions for quasi-linear PDEs involving the critical Sobolev and Hardy exponents. Trans. Amer. Math. Soc. 352, 5703-5743 (2000)

17. Guedda, M., Véron, L.: Quasilinear elliptic equations involving critical Sobolev exponents. Nonlinear Anal. TMA 13, 879-902 (1989)

18. Guo, Q.Q., Niu, P.C., Dou, J.B.: Multiplicity of solutions for singular semilinear elliptic equations with critical Hardy-Sobolev exponents. Appl. Anal. Discrete Math. 2, 158-174 (2008)

19. Han, P.G.: Quasilinear elliptic problems with critical exponents and Hardy terms. Nonlinear Anal. TMA $61,735-758(2005)$

20. Jannelli, E.: The role played by space dimension in elliptic critical problems. J. Differ. Equ. 156, 407-426 (1999)

21. Kang, D.S.: On the quasilinear elliptic problems with critical Sobolev-Hardy exponents and Hardy terms. Nonlinear Anal. TMA 68, 1973-1985 (2008)

22. Li, Y.Y., Guo, Q.Q., Niu, P.C.: Global compactness results for quasilinear elliptic problems with combined critical Sobolev-Hardy terms. Nonlinear Anal. TMA 74, 1445-1464 (2011)

23. Liang, S.H., Zhang, J.H.: Multiplicity of solutions for a class of quasilinear elliptic equation involving the critical Sobolev and Hardy exponents. Nonlinear Differ. Equ. Appl. 17, 55-67 (2010)

24. Pohozaev, S.I.: Eigenfunctions of the equation $\Delta u+\lambda f(u)=0$. Soviet Math. Dokl. 5, 1408-1411 (1965)

25. Ruiz, D., Willem, M.: Elliptic problems with critical exponents and Hardy potentials. J. Differ. Equ. 190, 524-538 (2003)

26. Silva, E., Xavier, M.: Multiplicity of solutions for quasilinear elliptic problems involving critical Sobolev exponents. Ann. IHP Anal. Nonlinéaire 20, 341-358 (2003)

27. Struwe, M.: A global compactness result for elliptic boundary value problems involving limiting nonlinearities. Math. Z. 187, 511-517 (1984) 